\title{
Al2O3 Nanopore Sensors for Single Molecule DNA Detection
}

\author{
B. M. Venkatesan, ${ }^{1,2}$ A.B. Shah, ${ }^{3,4}$ J.M. Zuo ${ }^{3,4}$ and R. Bashir ${ }^{1,2,45}$
}

${ }^{1}$ Department of Electrical and Computer Engineering, ${ }^{2}$ Micro and Nanotechnology Laboratory, ${ }^{3}$ Department of Materials Science and Engineering, ${ }^{4}$ Frederick Seitz Materials Research Laboratory, ${ }^{5}$ Department of Bioengineering, University of Illinois at Urbana Champaign, Urbana, Illinois

We report the development of a new solid-state, $\mathrm{Al}_{2} \mathrm{O}_{3}$ nanopore sensor with enhanced surface properties for the real-time detection and analysis of individual DNA molecules. The nanopore fabrication process involves first forming, free-standing, $45 \pm 5 \mathrm{~nm}$ thick $\mathrm{Al}_{2} \mathrm{O}_{3}$ membranes using standard micro-fabrication techniques. Next, a focused convergent electron beam from a field emission Transmission Electron Microscope (TEM) is used to decompositionally sputter single nanopores in these ultra-thin $\mathrm{Al}_{2} \mathrm{O}_{3}$ membranes. These nanopores exhibit excellent mechanical properties (high mechanical hardness, low stress) and state-of-the-art electrical performance (low noise, high signal-to-noise ratio), making them ideal for single molecule DNA analysis. ${ }^{[1]}$ Furthermore, we report drastic changes in the material properties of the nanopore during pore formation. Prolonged electron beam irradiation induces changes in the local stoichiometry and morphology of the pore from an amorphous, stoichiometric structure (O to $\mathrm{Al}$ ratio of 1.5) to a hetero-phase, crystalline structure with a nonstoichiometric $\mathrm{O}$ to $\mathrm{Al}$ ratio of $\sim 0.6$. Preferential phase transformations from $\gamma, \alpha, \kappa$ and $\delta-\mathrm{Al}_{2} \mathrm{O}_{3}$ to purely $\gamma$ and $\alpha$-phases are observed with increasing electron dose. Precise control over phase transformations in $\mathrm{Al}_{2} \mathrm{O}_{3}$ nanopore systems may provide a novel method to engineer surface charge at the nanopore/fluid interface. Metallization of the irradiated region is also observed, attributed to the preferential desorption of $\mathrm{O}$ and the aggregation of metallic Al clusters as confirmed through nanoarea electron diffraction and electron energy loss spectroscopy in the TEM. This in-situ metallization process may be applicable to the fabrication of nano-scale metallic contacts directly in the nanopore thereby enabling the electrical manipulation of surface charge and nanopore conductance.

The translocation of negatively charged dsDNA molecules through nanopores is conventionally done using two-terminal electrophoresis, resulting in characteristic blockades in the measured electrical current. DNA translocation experiments involving $\mathrm{Al}_{2} \mathrm{O}_{3}$ nanopores reveal average translocation velocities that are an order of magnitude less than that observed in $\mathrm{Si}_{3} \mathrm{~N}_{4}$ and $\mathrm{SiO}_{2}$ systems under similar conditions, attributed to strong DNA-nanopore interactions. At present, high DNA translocation velocities $(\sim 30 \text { bases } / \mu \mathrm{s})^{[2]}$ limit the utility of conventional $\mathrm{SiO}_{2}$ and $\mathrm{Si}_{3} \mathrm{~N}_{4}$ based nanopore technologies in high-end sensing applications such as single nucleotide detection. Thus, a nanopore architecture with an intrinsic ability to interact with DNA to reduce biomolecule transport velocity is highly desirable. Electron beam irradiated $\mathrm{Al}_{2} \mathrm{O}_{3}$ nanopore sensors provide such a capability. DNA-nanopore interactions are accredited to strong electrostatic binding events between anionic DNA and the positively charged nanopore surface, enhanced in $\mathrm{Al}_{2} \mathrm{O}_{3}$ due to high surface charge density and the nucleation of $\alpha, \gamma-\mathrm{Al}_{2} \mathrm{O}_{3}$ nanocrystallites. Our results confirm that nanopores formed in metal-oxide systems indeed provide a viable and highly functional alternative to conventional nanopore sensors. Such technologies find use in drug screening and medical applications involving label-free, real-time kinetic analysis of biomolecular interactions at the single molecule level. This nano-scale sensor may also be useful in studying the mechanisms driving biological processes including DNAprotein interactions, enzyme activity and cell signaling using gated, selective ion channels. 


\section{References}

[1] B. M. Venkatesan, B. Dorvel, S. Yemenicioglu, N. Watkins, I. Petrov, R. Bashir, Advanced Materials 2009, 21, 2771 .

[2] P. Chen, J. Gu, E. Brandin, Y.-R. Kim, Q. Wang, D. Branton, Nano Letters 2004, 4, 2293.
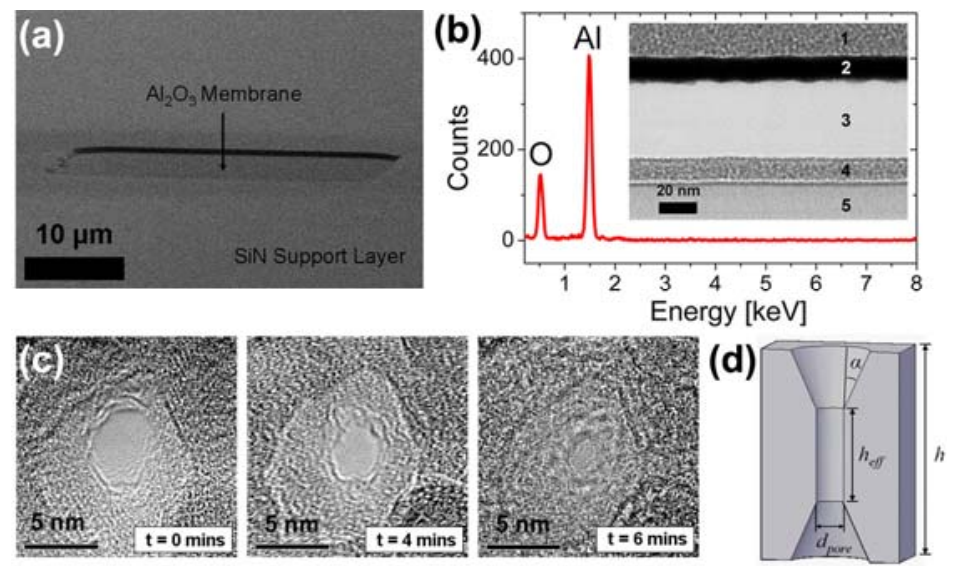

Figure 1 (a) Free-standing micro-fabricated $\mathrm{Al}_{2} \mathrm{O}_{3}$ membrane (b) EDS confirmed presence of only $\mathrm{Al}$ and $\mathrm{O}$ in the membrane (inset) cross sectional TEM of 45nm thick membrane (region 3) (c) Controlled nanopore contraction using TEM (d) Symmetric nanopore structure, resolved using energy filtered TEM and conductance measurements.
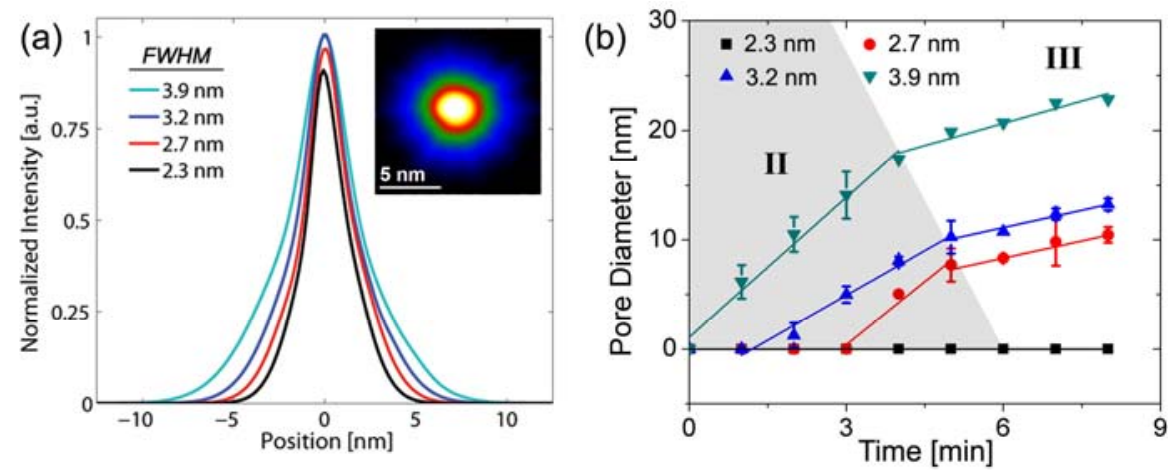

Figure 2 (a) Intensity profiles of the focused electron probes used for nanopore formation normalized with respect to peak intensity of the $3.9 \mathrm{~nm}$ probe (Inset) Intensity distribution of a $3.2 \mathrm{~nm}$ probe (b) Nanopore sputtering kinetics (pore diameter vs. time) for the four probes examined. Distinct expansion rates were observed delineating the three stages of pore formation, I Pore Nucleation (not shown), II Rapid Expansion and III Controlled Growth.
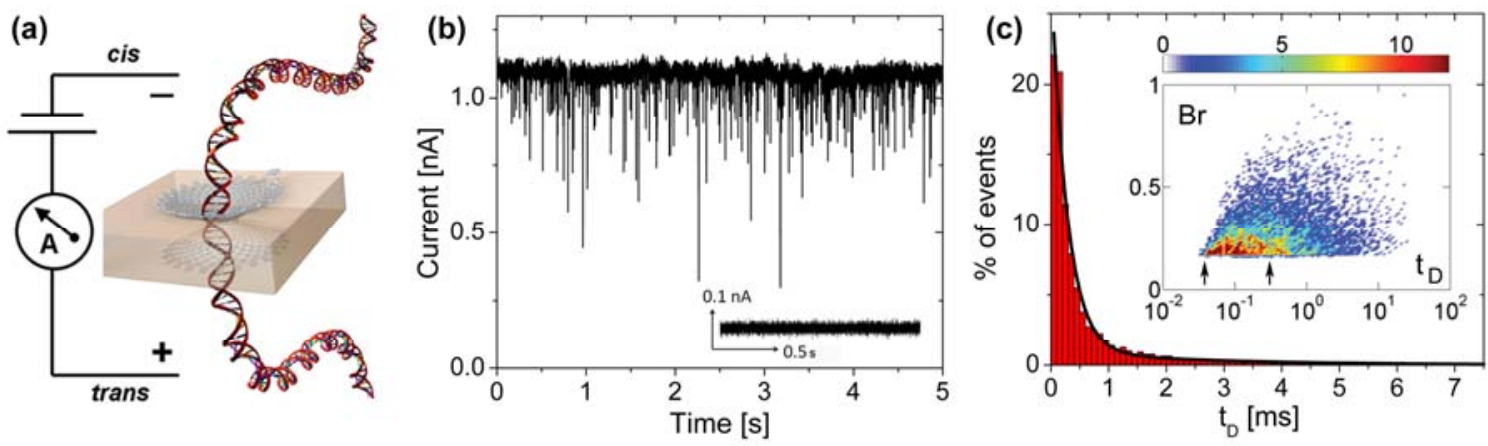

Figure 3 (a) Schematic of DNA translocation through an $\mathrm{Al}_{2} \mathrm{O}_{3}$ nanopore (b) Typical current traces from a $7 \mathrm{~nm}$ nanocrystalline $\mathrm{Al}_{2} \mathrm{O}_{3}$ pore after the addition of $5 \mathrm{kbp}$ dsDNA at $500 \mathrm{mV}$. Distinct downward current blockades are observed (Insets) No blockades are seen prior to the introduction of dsDNA (c) Dwell time histograms show a biexponential fit indicating the two types of polymer transport, fast translocation governed by polymer hydrodynamics and slow translocation regulated by DNA-pore interactions (Inset) Blockage Ratio $\left(\mathrm{B}_{\mathrm{r}}\right)$ vs. Dwell time $\left(\mathrm{t}_{\mathrm{D}}\right)$ [ms]. 